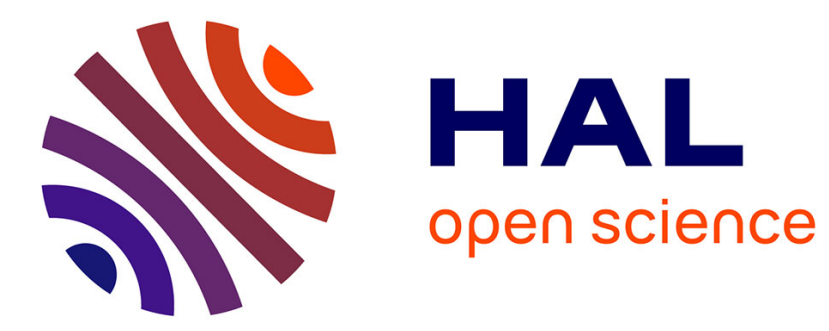

\title{
Softcast with per-carrier power-constrained channels
} Shuo Zheng, Marc Antonini, Marco Cagnazzo, Lorenzo Guerrieri, Michel Kieffer, Irina Delia Nemoianu, Roger Samy, Bo Zhang

\section{To cite this version:}

Shuo Zheng, Marc Antonini, Marco Cagnazzo, Lorenzo Guerrieri, Michel Kieffer, et al.. Softcast with per-carrier power-constrained channels. IEEE International conference on Image Processing, IEEE, Sep 2016, Phoenix, AZ, United States. pp.2122-2126, 10.1109/ICIP.2016.7532733 . hal-01424906

\section{HAL Id: hal-01424906 \\ https://hal-centralesupelec.archives-ouvertes.fr/hal-01424906}

Submitted on 6 Jan 2017

HAL is a multi-disciplinary open access archive for the deposit and dissemination of scientific research documents, whether they are published or not. The documents may come from teaching and research institutions in France or abroad, or from public or private research centers.
L'archive ouverte pluridisciplinaire HAL, est destinée au dépôt et à la diffusion de documents scientifiques de niveau recherche, publiés ou non, émanant des établissements d'enseignement et de recherche français ou étrangers, des laboratoires publics ou privés. 


\title{
SOFTCAST WITH PER-CARRIER POWER-CONSTRAINED CHANNELS
}

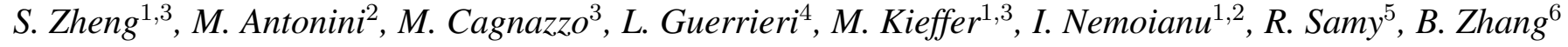 \\ ${ }^{1}$ L2S, CNRS-CentraleSupelec-Univ Paris-Sud, 3 rue Joliot-Curie, 91192 Gif-sur-Yvette, France \\ ${ }^{2}$ I3S, Univ. Nice-Sophia Antipolis, CNRS, 2000, route des Lucioles, 06903 Sophia Antipolis, France \\ ${ }^{3}$ LTCI, CNRS, Télécom ParisTech, Université Paris-Saclay, 75013, Paris, France \\ ${ }^{4}$ STMicroelectronics S.r.l., Via Lavoratori Vittime del Col Du Mont 28, 11100 Aosta, Italy \\ ${ }^{5}$ SAGEMCOM, R\&T Direction, 250 Route de L'Empereur, 92838 Rueil-Malmaison Cedex, France \\ ${ }^{6}$ Formerly of STMicroelectronics S.r.l.
}

\begin{abstract}
This paper considers the Softcast joint source-channel video coding scheme for data transmission over parallel channels with different power constraints and noise characteristics, typical in DSL or PLT channels. To minimize the mean square error at receiver, an optimal precoding matrix design problem has to be solved, which requires the solution of an inverse eigenvalue problem. Such solution is taken from the MIMO channel precoder design literature. Alternative suboptimal precoding matrices are also proposed and analyzed, showing the efficiency of the optimal precoding matrix within Softcast, which provides gains increasing with the encoded video quality.
\end{abstract}

Index Terms - Joint source-channel coding, Softcast, Precoding matrices, PLT channels

\section{INTRODUCTION}

A huge research effort has been devoted to design video coding and transmission systems to get the best received video quality for a given amount of channel resources. This is of paramount importance for cellular broadcasting, where channel conditions may be varying with time and among receivers, or for multimedia transmission in mobile ad-hoc networks, but also for communication over wired channels, such as DSL or powerline telecommunication (PLT) channels. Traditional solutions for these use-cases consist in hybrid video coding (H.264/AVC or HEVC $[2,3]$ ) or scalable video coding [4] over a suitable network protocol. However, in the first case the source encoding is performed without knowing the actual channel characteristics, causing channel underuse or digital cliff; in the second, even if the transmission rate can be adapted, each additional coding layer significantly reduces the performance of the system.

Joint source-channel video coding (JSCVC) has the potential for dramatically improve the quality of the received video in such challenging conditions, as demonstrated recently by breaking-through systems such as Softcast and its variants [5-7]. Reminiscent of hybrid digital-analog video coding ideas proposed by [8,9], Softcast is a JSCVC scheme that encodes the video content with linear-only operators (such as a full-frame DCT and scaling). The original pixel luminance is transformed into numerical values that are sent on the channel with an extremely dense modulation. This can be done in such a way that high-quality channels only perturb the least significant bits of the numerical values, while low-quality channels still

This information is based upon ETSI STF477 working assumptions [1]. The views expressed do not necessarily represent the position of ETSI in this context. preserve the most significant bits. In Softcast, compression involves a full-GoP 3D-DCT transform and transformed coefficient selection, error protection is obtained by power allocation and resilience to packet loss is obtained by giving up temporal prediction and on the contrary ensuring that all packets contribute equally to the quality of the decoded video. The physical layer uses a classical OFDM framework. Detailed comparisons with H.264/AVC or SVC over 802.11 wireless networks show very clearly the advantages of Softcast [5]. Many efforts have been done to adapt the data generated by Softcast and by linear coders in general to various channel conditions, such as wideband AWGN channels [10], fading channels [11, 12], MIMO channels [13]. Nevertheless, all of these works consider a single constraint on the total transmission power when evaluating the optimal scaling factors for the blocks of transform coefficients (these blocks are called chunks). For some channels, such as DSL or PLT [14], OFDM is employed and the power constraint depends on the index of the subchannel. Similarly, for multi-antenna transmission, each antenna has its own power amplifier and power constraint. In such situations, the simple approach of using a single total power constraint may give suboptimal scaling factors and individual power constraints may not be satisfied. An adaptation of Softcast when noise characteristics depend on the considered subchannel but with total power constraint can be found in [15]. The chunks with the most energy are transmitted over the best channels after a proper scaling. This solution, however does not consider different power constraints on each subchannel.

This paper deals with the adaptation of Softcast-based video transmission scheme considering parallel subchannels with different power constraints. To minimize the receiver MSE, one has to find a precoding matrix that transforms the chunk coefficients, modeled as independent Gaussian sources with different variances, so that they match the per-carrier power constraints. After recalling the main principles of Softcast in Section 2, we will describe this optimization problem in Section 3, recall the solution proposed in [16] and rediscovered later in the context of MIMO communication by $[17,18]$. The main contributions of this paper consist in adapting the precoding problem to the Softcast scheme (allowing to find optimal power allocation solution) and in proposing suboptimal but simpler alternative solutions. A final contribution is a simulation-based validation of the various precoding schemes, considering the transmission of several HD videos over a realistic PLT channel model (Section 4).

\section{SOFTCAST}

The general architecture of Softcast transmitter and receiver is shown in Fig. 1. The input digital video signal undergoes a linear 
3D-DCT transform, consisting of a full-frame 2D-DCT followed by a temporal 1D-DCT on a Group of Pictures $(\mathrm{GoP})$ of $n_{\mathrm{P}}$ frames.

Softcast works independently GoP by GoP. After a GoP has been transformed the resulting coefficients are grouped into chunks. A chunk is a set of $n_{r} \times n_{c}$ spatial coefficients belonging to the same temporal subband. The $n_{\mathrm{C}}$ chunks are sorted according to their variance and only the first $n_{\mathrm{S}}$ of them can be sent, according to the bandwidth limitations of the channel (see also the next section). The map of the selected chunk is robustly transmitted (using, e.g., a strong FEC code) on the channel: since the number of chunks is relatively small, the rate overhead needed to robustly transmit the map can be neglected.

The selected chunks are scaled in order to minimize the reconstruction error at the decoder. In [5], a simple power-constrained AWGN channel is considered. These results are extended to several parallel channels with different gains in [15]. The next section considers an extension of this result to parallel subchannels with individual power constraints and different noise characteristics.

\section{SUBCHANNELS WITH DIFFERENT POWER CONSTRAINTS}

The $j$-th coefficient of each of the $n_{\mathrm{C}}$ chunks are assumed generated by a source vector $\mathbf{x} \in \mathbb{R}^{n_{\mathrm{C}}}$ such that $E(\mathbf{x})=\mathbf{0}$ and $E\left(\mathbf{x x}^{\mathrm{T}}\right)=$ $\Lambda=\operatorname{diag}\left(\lambda_{1}, \ldots, \lambda_{n_{\mathrm{C}}}\right)$. This vector has to be transmitted over a wideband channel split into $n_{\mathrm{SB}}$ parallel subchannels. The power constraint for the $i$-th subchannel is $P_{i}$. The noise vector $\mathbf{v}$ corrupting the data on the subchannels is assumed to be zero-mean Gaussian process $\mathbf{v}$ with $E\left(\mathbf{v} \mathbf{v}^{\mathrm{T}}\right)=N=\operatorname{diag}\left(\sigma_{1}^{2}, \ldots, \sigma_{n_{\mathrm{SB}}}^{2}\right)$. One has thus to determine an efficient way to transmit the selected chunks over the subchannels.

For that purpose, one will use the results introduced in [16,17], which studied the problem of determining 1) an optimal precoding matrix $G \in \mathbb{R}^{n_{\mathrm{SB}} \times n_{\mathrm{C}}}$ to transform the source vector $\mathrm{x}$ before transmission to get $\mathbf{y}=G \mathbf{x}$; and 2) a decoding matrix $H \in \mathbb{R}^{n_{\mathrm{C}} \times n_{\mathrm{SB}}}$ at receiver side to retrieve $\widehat{\mathbf{x}}=H(G \mathbf{x}+\mathbf{v})$ such that $G$ and $H$ minimize

$$
\varepsilon=\operatorname{tr}\left(E(\mathbf{x}-\widehat{\mathbf{x}})(\mathbf{x}-\widehat{\mathbf{x}})^{\mathrm{T}}\right)
$$

and satisfy the subchannel power constraints

$$
\left(G \Lambda G^{\mathrm{T}}\right)_{i, i}=P_{i}, i=1, \ldots, n_{\mathrm{SB}}
$$

The Lagrangian of this constrained optimization problem is

$$
\begin{aligned}
& \mathcal{L}\left(G, H, \gamma_{1}, \ldots, \gamma_{\mathrm{SB}}\right)= \\
& \quad \operatorname{tr}\left(\Lambda-H G \Lambda-\Lambda G^{T} H^{T}+H\left(G \Lambda G^{T}+N\right) H^{T}\right) \\
& \quad+\sum_{i=1}^{n_{\mathrm{SB}}} \gamma_{i}\left(\left(G \Lambda G^{\mathrm{T}}\right)_{i, i}-P_{i}\right) .
\end{aligned}
$$

Fixing the precoding matrix, $[16,17]$ obtained the optimum decoding matrix

$$
H=\Lambda G^{T}\left(G \Lambda G^{T}+N\right)^{-1} .
$$

Moreover, a necessary condition for optimality is $\left(H^{T} H+\Gamma\right) G=$ $H^{T}$ with $\Gamma=\operatorname{diag}\left(\gamma_{1}, \ldots, \gamma_{\mathrm{SB}}\right)$, the matrix of Lagrange multipliers.

Using the previous results and introducing

$$
G^{\prime}=N^{-1 / 2} G,
$$

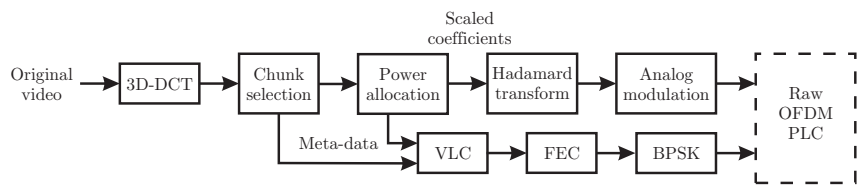

(a) Softcast Transmitter

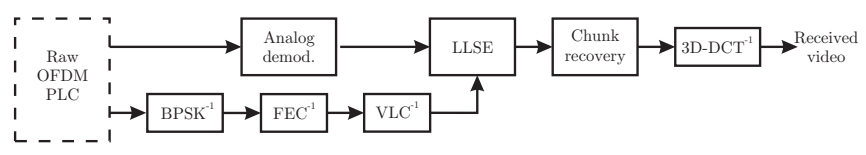

(b) Softcast receiver

Fig. 1: Softcast transmitter and receiver

(1) may be rewritten as

$$
\varepsilon=\operatorname{tr}\left(\Lambda-\Lambda G^{\prime T}\left(G^{\prime} \Lambda G^{T}+I\right)^{-1} G^{\prime} \Lambda\right)
$$

which has to be minimized with the constraints

$$
\left(N^{1 / 2} G^{\prime} \Lambda G^{\prime \mathrm{T}} N^{1 / 2}\right)_{i, i}=P_{i}, i=1, \ldots, n_{\mathrm{SB}}
$$

leading to

$$
G^{\prime} \Lambda G^{\prime \mathrm{T}}=\left(\begin{array}{cccc}
P_{1} / \sigma_{1}^{2} & * & * & * \\
* & P_{2} / \sigma_{2}^{2} & * & * \\
* & * & \ddots & * \\
* & * & * & P_{n_{\mathrm{SB}}} / \sigma_{n_{\mathrm{SB}}}^{2}
\end{array}\right)=P^{\prime} .
$$

When some $G^{\prime}$ minimizing (5) with the constraint (7) has been found, then $G=N^{1 / 2} G^{\prime}$ and the corresponding $H$ minimize (1) with the constraint (2). For this reason, one considers first the problem of finding the optimal precoding matrix $G^{\prime}$ with signal-to-noise constraints (7) that minimizes (5). One can thus define an equivalent channel with subchannel power constraints corresponding to the signal-to-noise ratios of the original subchannels and uncorrelated unit-variance noise components. Once a precoding matrix $G^{\prime}$ has been found for the equivalent channel, the matrix $G$ for the original channel may be deduced from (4).

In what follows, one assumes without loss of generality that $\lambda_{1} \geqslant \cdots \geqslant \lambda_{n_{\mathrm{C}}}$ and that $\sigma_{1}^{2} \leqslant \cdots \leqslant \sigma_{n_{\mathrm{SB}}}^{2}$.

\subsection{Optimal solution}

To minimize (5) with the constraint (7), [16] first consider the solution with the total power constraint of the equivalent channel

$$
P_{\mathrm{eq}}=\sum_{i=1}^{n_{\mathrm{SB}}} P_{i} / \sigma_{i}^{2}
$$

for which the optimal encoding matrix has the form

$$
G=\left(\begin{array}{cc}
\operatorname{diag}\left(g_{11}, \ldots, g_{n_{\mathrm{S}} n_{\mathrm{S}}}\right) & 0 \\
0 & 0
\end{array}\right)
$$

where $n_{\mathrm{S}} \leqslant \min \left(n_{\mathrm{SB}}, n_{\mathrm{C}}\right)$ is the largest integer satisfying

$$
\lambda_{n_{\mathrm{S}}} \geqslant \gamma, \sqrt{\gamma}=\frac{\sum_{i=1}^{n_{\mathrm{S}}} \sqrt{\lambda_{i}}}{P_{\mathrm{eq}}+n_{\mathrm{S}}} \text {, and } g_{i i}=\sqrt{\frac{\sqrt{\lambda_{i} / \gamma}-1}{\lambda_{i}}} \text {. }
$$




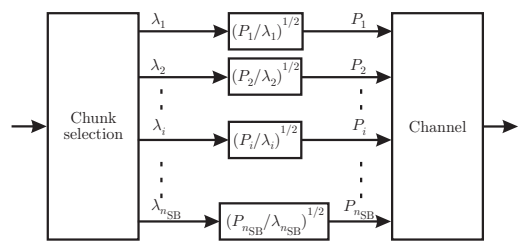

Fig. 2: Simple chunk scaling, assuming unit channel noise variance

When the noise components are uncorrelated and of equal characteristics, as is the case for the equivalent channel, [16] shows that any orthogonal transform matrix does not change optimality. Then, considering the diagonal matrix $D$ with the $g_{i i}$ s as diagonal elements and introducing the matrix $M=D \Lambda D^{\mathrm{T}}$, one has to find an orthogonal transformation matrix $Z$ such that $Z M Z^{\mathrm{T}}=P^{\prime}$. If such $Z$ exists,

$$
G^{\prime}=Z D
$$

Finding $Z$ requires to solve an inverse eigenvalue problem, since one has to determine the off-diagonal elements of $P^{\prime}$ that has prescribed eigenvalues given by the diagonal matrix $M$. A constructive solution based on majorization theory is proposed in $[16,17,19]$, provided that the $l$ largest diagonal elements of $M$ are larger than the sum of the $l$ corresponding diagonal elements of $P^{\prime}$ and $\operatorname{tr} M=\operatorname{tr} P^{\prime}$.

When the previous necessary condition is not satisfied, one considers a matrix of Lagrange multipliers of the form

$$
\Gamma=\left(\begin{array}{cc}
\gamma_{1} I_{1} & 0 \\
0 & \gamma_{2} I_{2}
\end{array}\right)
$$

where $I_{1}$ and $I_{2}$ are $p \times p$ and $\left(n_{\mathrm{SB}}-p\right) \times\left(n_{\mathrm{SB}}-p\right)$ identity matrices. The optimal matrix $G^{\prime}$ has then the form

$$
G^{\prime}=\left(\begin{array}{cc}
Z_{1} D_{1} & 0 \\
0 & Z_{2} D_{2}
\end{array}\right)
$$

where $D_{1}, D_{2}$ and $Z_{1}, Z_{2}$ are obtained solving two total power constraint optimization problems and using two numerical inverse Jacobi methods, see [17] for more details.

Nevertheless, the computed matrix $G^{\prime}$ (or $G$ ) has to be transmitted to the receiver to allow decoding. As can be seen in $[16,17]$, $G$ is a scaled product of rotation matrices (due to the inverse Jacobi method), which may be efficiently encoded. Nevertheless, for large $n_{\mathrm{SB}}$, the evaluation complexity for $G$ may become significant.

\subsection{Alternative solutions}

Two suboptimal solutions are now proposed, in which the transform matrix $G$ still satisfies (2), but may be obtained in a much easier way. In what follows, one assumes without loss of generality that the chunks and the subchannels are ordered with decreasing variance and with decreasing signal-to-noise ratios (SNR). Moreover, one considers only the equivalent channel.

In the first solution, called Simple Chunk Scaling (SCS), the chunk of largest variance is transmitted over the subchannel with the best SNR. The chunk with the second largest variance is sent over the subchannel with the second best SNR, etc., see Fig. 2. To fit the power constraint, the coefficients of the $i$-th chunk are multiplied by $g_{\mathrm{SCS}, i}=\sqrt{P_{i} /\left(\lambda_{i}\right)}$.

The second solution, called Simple Chunk Scaling with Hadamard Transform (SCSHT), considers the optimal chunk scaling (10) obtained considering the total power constraint (8). The scaled chunks are then Hadamard-transformed to get transformed chunks of similar power $P_{\mathrm{eq}} / n_{\mathrm{SB}}$, see Fig. 3. The scaled chunks

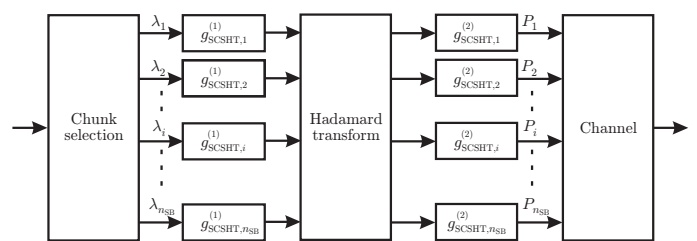

Fig. 3: Simple chunk scaling with Hadamard transform, assuming unit channel noise variance

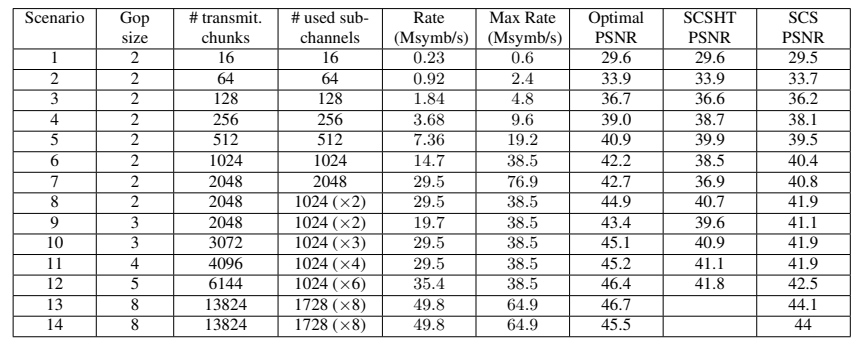

Table 1: Simulation results for Komono1, average PSNRs are in dB

are then rescaled to fit the individual channel power constraints. The two scaling factors are then $g_{\mathrm{SCSHT}, i}^{(1)}=g_{i i}, i=1 \ldots n_{s}$, $g_{\mathrm{SCSHT}, i}^{(1)}=0, i=n_{s+1} \ldots n_{\mathrm{SB}}$ and $g_{\mathrm{SCSHT}, i}^{(2)}=\sqrt{n_{\mathrm{SB}} P_{i} / P_{\mathrm{eq}}}$, where $g_{i i}$ is given by (10). This solution is close to the original Softcast scheme proposed in [5], where a total power constraint is considered.

\section{EXPERIMENTAL RESULTS}

In order to perform simulations, we first used the luminance of the Kimonol video sequence from the HEVC test set. It is a HD video sequence (resolution: $1920 \times 1080$ ), with 24 frames per second. Chuncks of $30 \times 40$ coefficients are used.

An in-home power line channel is considered for transmission. The considered frequency range is from $1.8 \mathrm{MHz}$ to $86.13 \mathrm{MHz}$, which is the same range considered by the HomePlug Alliance in the last generation specification HomePlug AV2 [14]. The spacing between subcarriers is $24.414 \mathrm{kHz}$. In OFDM-based PLT systems like AV2, typically SNRs per subchannel are available. Each subchannel is assumed to be modeled accurately by an AWGN channel. An example of the individual subchannel SNR constraints are represented in Fig. 4, which relates to a bad SISO link from ETSI STF 477 database [1].

Considering a Homeplug AV2-type physical layer adapted to Softcast, in which root-raised-cosine Nyquist filters with 30\% rolloff are used, one obtains a per-subchannel transmission rate of $37.56 \times 10^{3}$ real-valued symbols per second. Remember that Softcast employs very high-density or analog constellations.

The various chunk scaling schemes are compared considering the average received video Peak Signal-to-Noise (PSNR) between the original video and the reconstructed video. In what follows, one considers noise with equal unit variance for each subcarrier.

Several transmission configurations are considered, according to the GoP size, the number of transmitted chunks and the number of subchannels used. The latter determines the maximum transmission rate and the maximum number of chunks that may be transmitted per GoP.

Results are provided in Table 1. Besides the scenario index, the first column is the GoP size; the second is the number of transmitted 


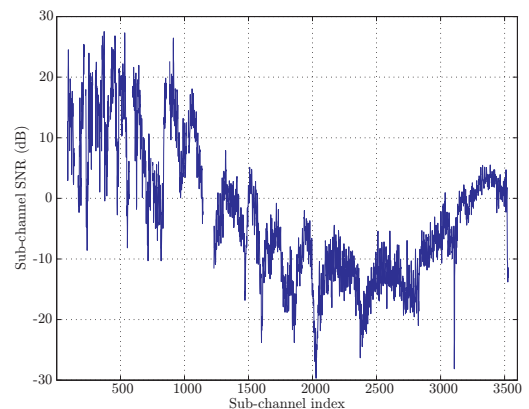

Fig. 4: SNR as a function of the subcarrier index for the considered PLT channel

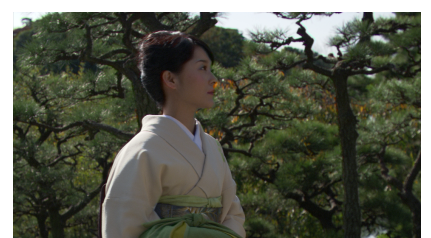

(a) Original

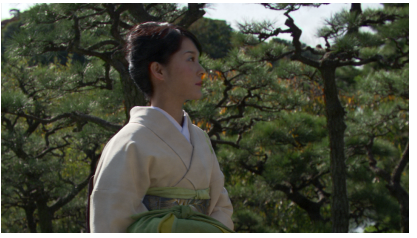

(c) SCS, PSNR-Y = 42.1dB

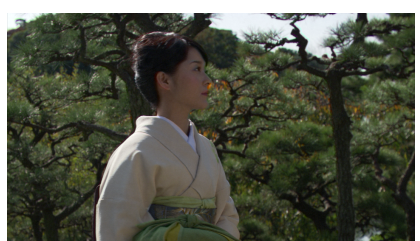

(b) Optimal, PSNR-Y $=45.9 \mathrm{~dB}$

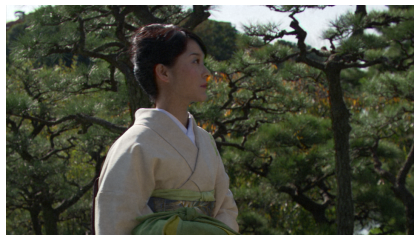

(d) SCS-HT, PSNR-Y $=41.6 \mathrm{~dB}$
Fig. 5: Comparison of the proposed schemes on Frame 140 of Kimono1 using Scenario 8; zoom the face to see the differences.

chunks; the third is the number of subchannels used; the fourth and fifth are the corresponding rates. Columns 6 to 8 report the resulting PSNRs for the three considered power allocation strategies.

We first remark that the optimum precoding matrix provides always better results than the other ones. In particular, for a GoP size equal to 2, if we transmit more chunks, the optimal strategy has increasing gains, up to $3 \mathrm{~dB}$ with respect to SCS and $4.3 \mathrm{~dB}$ with respect to the SCSHT (plain Softcast). Considering the two last rows for GoP size 2, if we separate the transmission in two time slots and we always use the best 1024 subchannels, we obtain even better performance compared with using all subchannels and transmitting them in one time slot.

If we increase the GoP size but keep the number of chunks and number of subchannels unchanged, the PSNR will be worse because there are not enough chunks to represent the images. On the other hand, if we increase the number of chunks, the PSNR is increased accordingly. In any case, the proposed strategy gains several dBs with respect to the plain Softcast, see also Fig. 5.

We consider also a reduced complexity version of the proposed strategy, whose results are shown in the last row of Table 1. The reduced-complexity variant consists in gathering the chunks in groups of $s$ chunks. Then, the variance of the group is used in the optimization problem. This divides the dimensionality of the problem by the number of groups, but the resulting precoding matrix is suboptimal.

In Scenario 13 and 14, GoPs of 8 frames are considered. A total of 13824 chunks per GoP has to be transmitted. In Scenario 13, these chunks are transmitted in 8 time slots. For each time slot, 1728

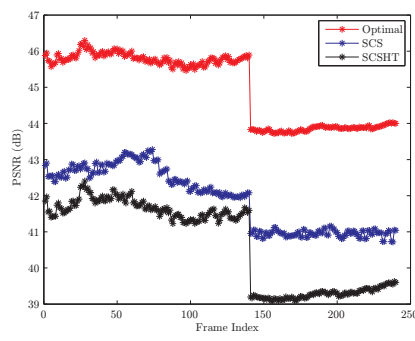

(a) Kimono1

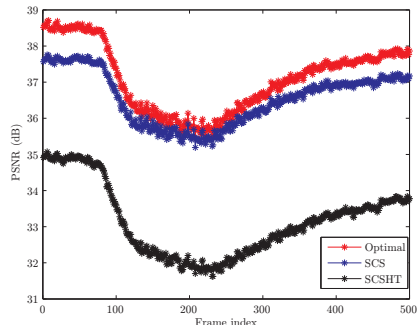

(c) DucksTakeOff

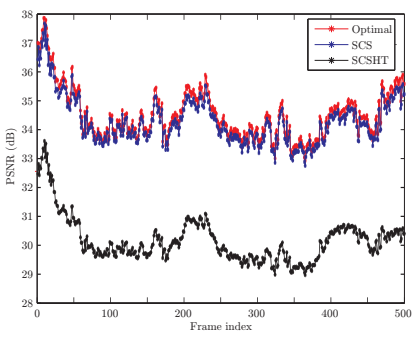

(b) ParkJoy

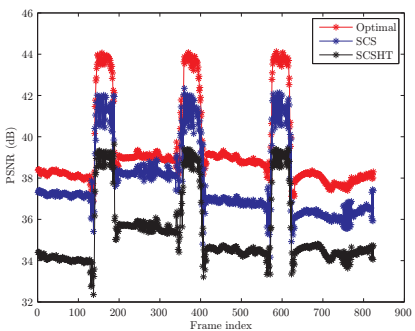

(d) Life
Fig. 6: PSNR as a function of time for Scenario 8.

\begin{tabular}{|c|c|c|c|c|c|c|}
\hline & \multicolumn{3}{|c|}{ PSNR Scenario 7 } & \multicolumn{3}{c|}{ PSNR Scenario 8 } \\
\hline Video & Optimal & SCSHT & SCS & Optimal & SCSHT & SCS \\
\hline Kimono1 & 42.7 & 36.9 & 40.8 & 44.9 & 40.7 & 41.9 \\
\hline ParkJoy & 30.7 & 26.0 & 30.6 & 34.5 & 30.1 & 34.2 \\
\hline DucksTakeOff & 34.3 & 29.3 & 34.1 & 37.1 & 33.1 & 36.5 \\
\hline Life & 36.2 & 31.4 & 35.5 & 39.3 & 35.3 & 37.8 \\
\hline
\end{tabular}

Table 2: Average PSNRs (in $\mathrm{dB}$ ) over all frames for HD videos

chunks are sent over the best 1728 subchannels. In Scenario 14, the sub-optimal strategy is used and in each time slot, the 1728 chunks are split in two parts of 864 chunks each, and the same is done for the subchannels. Two optimal predocing matrices are then designed for the first and the last 864 chunks and first and last 864 channels. Scenario 14 shows that this strategy results in a PSNR decrease of $1.2 \mathrm{~dB}$ compared to Scenario 13, but on the other hand the precoding matrix design algorithm takes only $57 \%$ of the time of the original one. We observe that the reduced-complexity strategy provides an alternative trade-off, and that it is still better than references.

In a second set of simulations, three additional HD video sequences are considered: DuckTakeOff, ParkJoy, and Life. Table 2 presents the resulting PSNRs averaged over all frames of the video sequences for Scenario 7 and 8. Fig. 6 shows the evolution with time of the PSNRs obtained with the various solutions. The highest benefits provided by the optimal precoding matrix are for the parts of all video sequences which have the best quality.

\section{CONCLUSIONS}

In this paper we consider a Softcast video coding and transmission scheme with a power allocation when multiple channels with different power constraints and noise characteristics have to be considered. An equivalent channel where the different power constraints are translated into signal-to-noise constraints is considered. The problem is then formulated as an inverse eigenvalues problem. Using an existing iterative algorithm for the inverse eigenvalues problem, we provide an optimal power allocation strategy that has better performance than alternative strategies that can be found in the stateof-the-art. Future work may address the complexity reduction of the proposed strategy. 


\section{REFERENCES}

[1] ETSI TR 103343 V1.1.1, "Powerline telecommunications (PLT); powerline HDMI ${ }^{\circledR}$ analysis for very short range link HD and UHD applications," Tech. Rep., ETSI, Dec. 2015.

[2] T. Wiegand, G. J. Sullivan, G. Bjøntegaard, and A. Luthra, "Overview of the H.264/AVC video coding standard," IEEE Trans. on Circuits and Systems for Video Technology, vol. 13(7), pp. 560-576, 2003.

[3] G. J. Sullivan, J.-R. Ohm, W.-J. Han, and T. Wiegand, "Overview of the high efficiency video coding (HEVC) standard," IEEE Trans. on Circuits Syst. Video Technol., vol. 22, no. 12, pp. 1649-1668, 2012.

[4] H. Schwarz, D. Marpe, and T. Wiegand, "Overview of the scalable video coding extension of the H.264/AVC standard," IEEE Trans. on Circuits Syst. Video Technol., vol. 17, no. 9, pp. 1103-1120, Sept. 2007.

[5] S. Jakubczak and D. Katabi, "Softcast: one-size-fits-all wireless video," in Proceedings of the ACM SIGCOMM 2010 conference, New York, NY, USA, 2010, SIGCOMM '10, pp. 449450, ACM.

[6] Song Zhihai, Xiong Ruiqin, Ma Siwei, Fan Xiaopeng, and Gao Wen, "Layered image/video softcast with hybrid digital-analog transmission for robust wireless visual communication," in Multimedia and Expo (ICME), 2014 IEEE International Conference, 2014.

[7] Xiong. Ruiqin, Wu. Feng, Fan. Xiaopeng, Luo. Chong, Ma. Siwei, and Gao. Wen, "Power-distortion optimization for wireless image/video softcast by transform coefficients energy modeling with adaptive chunk division," in Visual Communications and Image Processing (VCIP), 2013.

[8] M.O. Polley, S.J. Wee, and W.F. Schreiber, "Hybrid channel coding for multiresolution hdtv terrestrial broadcasting," in Proc. IEEE International ConferenceImage Processing, Nov 1994, vol. 1, pp. 243-247.

[9] W. Schreiber, "Advanced television systems for terrestrial broadcasting: Some problems and some proposed solutions," Proceedings of the IEEE, vol. 83, no. 6, pp. 958-981, Jun 1995.

[10] S. Jakubczak, J.Z. Sun, D. Katabi, and V.K. Goyal, "Performance regimes of uncoded linear communications over awgn channels," in Proc. 45th Annual Conference on Information Sciences and Systems (CISS), March 2011, pp. 1-6.

[11] Z. Zhang, D. Liu, X. Ma, and X. Wang, "Ecast: An enhanced video transmission design for wireless multicast systems over fading channels," IEEE Systems Journal, vol. PP, no. 99, pp. 1-12, 2015.

[12] Fan Zhang, Anhong Wang, Haidong Wang, Suyue Li, and Xiaoli Ma, "Channel-aware video softcast scheme," in Proc. IEEE China Summit and International Conference on Signal and Information Processing (ChinaSIP), July 2015, pp. 578581.

[13] Xiao Lin Liu, Wenjun Hu, Chong Luo, and Feng Wu, "Compressive image broadcasting in MIMO systems with receiver antenna heterogeneity," Signal Processing: Image Communication, vol. 29, no. 3, pp. 361-374, 2014.

[14] L. Yonge, J. Abad, K. Afkhamie, L. Guerrieri, S. Katar, H. Lioe, P. Pagani, R. Riva, D. M. Schneider, and A. Schwager, "An overview of the homeplug AV2 technology," Journal of Electrical and Computer Engineering, p. 20, 2013.
[15] Fan Zhang, Anhong Wang, Haidong Wang, Suyue Li, and Xiaoli Ma, "Channel-aware video softcast scheme," in Signal and Information Processing (ChinaSIP), 2015 IEEE China Summit and International Conference, 2015, pp. 578-581.

[16] K. H. Lee and D. P. Petersen, "Optimal linear coding for vector channels," IEEE Trans. On Communications, vol. 24, no. 12, pp. 1283-1290, 1976.

[17] D. P. Palomar, M. A. Lagunas, and J. M. Cioffi, "Optimum linear joint transmit-receive processing for MIMO channels with qos constraints," IEEE trans. Signal Processing, vol. 52, no. 5, pp. 1179-1197, may 2004.

[18] D. P. Palomar and Y. Jiang, MIMO transceiver design via majorization theory, vol. 3 of Foundations and trends in communications and information theory, Now, 2006.

[19] P. Viswanath and V. Anantharam, "Optimal sequences and sum capacity of synchronous CDMA systems," IEEE trans. Information Theory, vol. 45, no. 6, pp. 1984-1991, September 1999. 